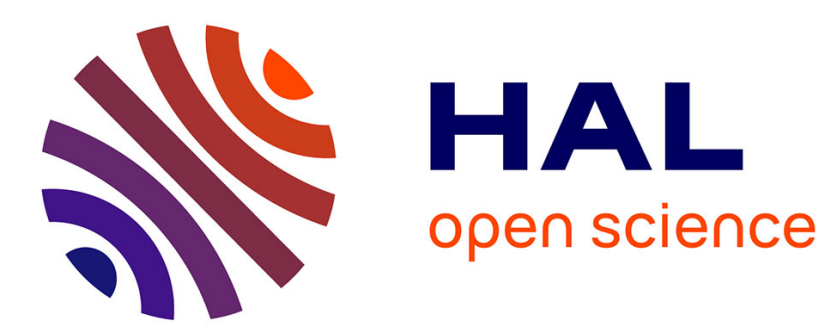

\title{
Microdéformation associée à la création de doubles décrochements dans l'aluminium
}

C. Esnouf, Gilbert Fantozzi

\section{To cite this version:}

C. Esnouf, Gilbert Fantozzi. Microdéformation associée à la création de doubles décrochements dans l'aluminium. Revue de Physique Appliquée, 1977, 12 (1), pp.31-39. 10.1051/rphysap:0197700120103100 . jpa-00244117

HAL Id: jpa-00244117 https://hal.science/jpa-00244117

Submitted on 1 Jan 1977

HAL is a multi-disciplinary open access archive for the deposit and dissemination of scientific research documents, whether they are published or not. The documents may come from teaching and research institutions in France or abroad, or from public or private research centers.
L'archive ouverte pluridisciplinaire HAL, est destinée au dépôt et à la diffusion de documents scientifiques de niveau recherche, publiés ou non, émanant des établissements d'enseignement et de recherche français ou étrangers, des laboratoires publics ou privés. 
Classification

Physics Abstracts

$7.222-7.240$

\title{
MICRODÉFORMATION ASSOCIÉE A LA CRÉATION DE DOUBLES DÉCROCHEMENTS DANS L'ALUMINIUM $\left(^{*}\right)$
}

\author{
C. ESNOUF $(* *)$ et G. FANTOZZI $(* *)$
}

I. N. S. A., Bâtiment 502, 69621 Villeurbanne, France

(Reçu le 3 mars 1976, révisé le 29 septembre 1976, accepté le 11 octobre 1976)

\begin{abstract}
Résumé. - Nous avons étudié le comportement d'un aluminium soumis à une faible contrainte statique. Nous observons dans le domaine de température du pic de Bordoni, une microdéformation anélastique quasi-réversible. Cette microdéformation peut être associée à la création thermiquement activée de doubles décrochements. L'énergie et le volume d'activation déterminés à partir des résultats expérimentaux sont tout à fait compatibles avec un tel mécanisme. En outre, notre hypothèse est confirmée par l'étude de la variation de la limite anélastique avec la température et la vitesse de déformation.
\end{abstract}

Abstract. - We have studied the behaviour of an aluminium submitted to a low bias stress. In the temperature range of the Bordoni peak, an anelastic and reversible microdeformation is observed. That this microdeformation can be attributed to thermally activated double-kink formation is supported by measurements of activation energy and volume, as well as by studies of the variation of the anelastic limit as a function of temperature and strain rate.

1. Introduction. - Après déformation plastique, le frottement intérieur des métaux c. f. c., mesuré aux basses températures $(10-150 \mathrm{~K})$, est généralement attribué aux propriétés intrinsèques des dislocations [1]. En particulier, il apparaît un pic dit pic de Bordoni, que de nombreux auteurs associent à la création thermiquement activée de doubles décrochements [2]. On en déduit ainsi une valeur de la contrainte de Peierls $\sigma_{\mathrm{p}}$ qui est de l'ordre de $5 \times 10^{-4} \mathrm{G}, \mathrm{G}$ étant le module de cisaillement.

La plasticité des métaux c. f. c. à basse température, devrait donc être contrôlée par l'existence de cette contrainte de Peierls-Nabarro. Or l'étude de la variation avec la température de la limite élastique macroscopique $\left({ }^{1}\right)$ semble contradictoire avec cette hypothèse [3] : d'une part les matériaux $c$. f. c. se déforment à basse température pour des contraintes inférieures à $\sigma_{\mathrm{p}}$, d'autre part le volume d'activation correspondant au mécanisme de déformation est nettement plus important que celui associé au mécanisme de création de paires de décrochements (environ 10 fois plus grand). La plupart des auteurs attribuent cette variation de la limite élastique au mécanisme d'intersection des dislocations $[4,5,6]$.

(*) Cet article recouvre, en partie, le travail d'une thèse qui sera soutenue par C. Esnouf à l'Université Claude-Bernard de Lyon en 1976, $\mathrm{n}^{\mathrm{o}}$ d'enregistrement du C. N. R. S. : A. O. 12.029.

$\left({ }^{* *}\right)$ Groupe d'études de métallurgie physique et de physique des matériaux, équipe de recherche associée au C. N. R. S. sous le numéro 463 .
Afin d'essayer de relever cette contradiction, il nous a paru intéressant d'étudier le comportement d'un aluminium sous l'effet d'une faible contrainte appliquée. En effet, avec l'application d'une contrainte statique, un stade de microdéformation $\left({ }^{1}\right)$ doit apparaître dans le même domaine de température que celui du pic de Bordoni, microdéformation qui serait due au franchissement thermiquement activé des barrières de Peierls par les dislocations parallèles ou presque aux vallées de Peierls. L'énergie d'activation de ce processus doit être égale à l'énergie nécessaire pour créer un double décrochement (de l'ordre de $0,1 \mathrm{eV}$ ), le volume d'activation étant de l'ordre de grandeur de l'aire balayée lors de la création d'une paire de décrochements multipliée par le vecteur de Burgers $\left(\simeq 100 b^{3}\right)$.

De plus, la variation avec la température de la limite anélastique $\left({ }^{1}\right)$ d'un échantillon déformé doit être compatible avec le mécanisme de création de doubles décrochements. Ainsi les valeurs de l'énergie et du volume d'activation déduites de ces mesures doivent être proches de $0,1 \mathrm{eV}$ et de $100 b^{3}$ respectivement.

(1) On définit la limite anélastique comme étant la contrainte à partir de laquelle une faible déformation résiduelle (de l'ordre de 10-6) subsiste après avoir effectué un cycle de contrainte. La limite élastique macroscopique (ou macroélastique) correspond à des déformations plastiques de l'ordre de $10^{-3}$. Toute déformation produite par des contraintes inférieures à la limite macroélastique est appelée microdéformation $[10,11]$. 
La comparaison des résultats obtenus par les deux techniques (microdéformation et mesure de limite anélastique) permet ainsi de préciser les mécanismes mis en jeu lors de la microdéformation.

2. Méthode expérimentale. - 2.1 InSTALLATION DE MESURE. - Les mesures ont été effectuées à l'aide d'un pendule de torsion inversé oscillant à l'hertz asservi à amplitude constante $\left(\varepsilon_{\mathrm{m}}=2 \times 10^{-5}\right)$ permettant l'enregistrement du frottement intérieur et de la période d'oscillation entre 6 et $500 \mathrm{~K}$. En outre, un couple de torsion peut être superposé à la contrainte de mesure en utilisant le champ magnétique créé par deux bobines parcourues par un courant constant. On suit la microdéformation de l'éprouvette sous l'action de ce couple en enregistrant le déplacement de l'ensemble cellulaire. On arrive ainsi à détecter une microdéformation de l'ordre de $10^{-6}$.

2.2 MAtÉRIAUX UTILISÉS. - Nous avons utilisé un aluminium AIAG de pureté 99,999\%. Les éprouvettes sont recuites après laminage par un maintien de une heure à $500 \mathrm{~K}$. Les échantillons utilisés pour le microfluage sont irradiés dans les boucles à azote liquide dans la pile piscine Mélusine à neutrons rapides du C. E. N. Grenoble. Ces éprouvettes sont montées à $80 \mathrm{~K}$ sur l'ensemble pendulaire puis écrouies par torsion soit à $10 \mathrm{~K}$, soit à $80 \mathrm{~K}$.

Les échantillons utilisés pour la détermination de la limite anélastique sont simplement montés sur le pendule à la température ambiante puis écrouis par torsion à $10 \mathrm{~K}$.

2.3 Traitements DeS matériauX. - Nous avons effectué deux traitements préalables :

Traitement 1. - L'aluminium est irradié aux neu. trons rapides à $77 \mathrm{~K}$ à une dose de $10^{18} \mathrm{~N} / \mathrm{cm}^{2}$, déformé par torsion de $0,5 \%$ ou $0,75 \%$ et recuit à $273 \mathrm{~K}$. L'irradiation aux neutrons permet d'introduire une concentration importante de défauts ponctuels.

La figure 1 montre les spectres de frottement intérieur obtenus avant et après le recuit à $273 \mathrm{~K}$. Directement après écrouissage (courbe $A$ ), on observe un frottement intérieur faible à cause de l'ancrage important des lignes de dislocations par les défauts ponctuels ou amas créés par l'irradiation. Après recuit à $220 \mathrm{~K}$ (courbe $B$ ) le spectre de frottement intérieur est essentiellement constitué du pic de Bordoni $\mathbf{B}_{2}$ (situé vers $100 \mathrm{~K}$ ) dont l'amplitude reste faible (le pic de Niblett et Wilks $B_{1}$ qui apparaît vers $65 \mathrm{~K}$ est quasi inexistant). Le recuit à $273 \mathrm{~K}$ (courbe $C$ ) entraîne une migration importante des défauts lacunaires provoquant une réorganisation du réseau de dislocations et une diminution des contraintes internes [7], le pic de Bordoni étant maximum. En effet, l'observation au microscope électronique d'échantillons déformés non irradiés met en évidence une

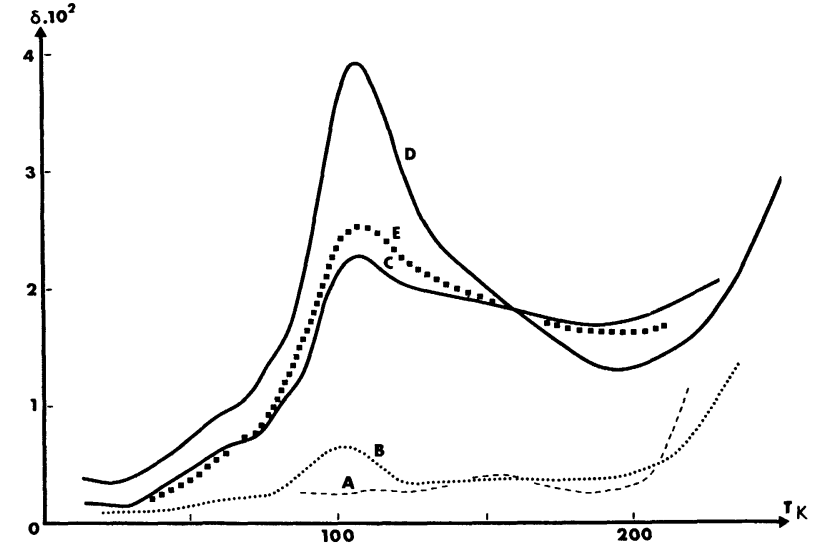

FIG. 1. - Spectres de frottement intérieur obtenus sur un aluminium AIAG irradié à $77 \mathrm{~K}$ à la dose de $10^{18} \mathrm{~N} / \mathrm{cm}^{2}$ puis écroui de $0,7 \%$ à $80 \mathrm{~K}$. $A$ : directement après la déformation plastique ; $B$ : après recuit à $220 \mathrm{~K} ; C$ : après recuit à $273 \mathrm{~K}$; $D$ : même état mais une contrainte statique de $10^{-4} \mathrm{G}$ est superposée à la contrainte de mesure ; $E$ : après suppression de la contrainte statique.

configuration de dislocations enchevêtrées et essentiellement mixtes. Après irradiation et déformation, le recuit à $273 \mathrm{~K}$ diminue la densité totale de dislocations mais fait apparaître de longues dislocations rectilignes de caractère essentiellement vis. Une telle répartition des dislocations a pour conséquence l'existence de faibles contraintes internes: en effet, le spectre de frottement intérieur obtenu après ce traitement est caractéristique d'un tel état, la condition de Paré $\left({ }^{2}\right)$ nécessaire à l'obtention d'un processus de relaxation important $[2,8]$ n'est plus satisfaite et il apparaît ainsi un maximum diffus vers $115 \mathrm{~K}$. En outre, l'amplitude importante du frottement intérieur obtenue par ce traitement montre que la majorité des dislocations participent au mécanisme de relaxation. L'application d'une contrainte statique de $10^{-4} \mathrm{G}$ rétablit la condition de Paré et un pic de Bordoni bien défini apparaît (courbe $D$ ). Après suppression de cette contrainte, on retrouve presque l'état initial (courbe $E$ ); on peut donc considérer qu'une contrainte statique de l'ordre de $10^{-4} \mathrm{G}$ ne perturbe pratiquement pas l'échantillon. Ainsi, le traitement décrit précédemment, permet d'obtenir :

- de longues dislocations en majorité rectilignes,

- de faibles contraintes internes,

- une limite anélastique à $10 \mathrm{~K}$ supérieure à $10^{-4} \mathrm{G}$.

Cet état est idéal pour réaliser les expériences de microdéformation.

(2) La condition de Paré est la condition nécessaire pour obtenir deux configurations d'une même dislocation également probables. Cette condition peut être remplie à l'aide de contraintes internes ou de contraintes appliquées. 
Traitement 2. - L'aluminium est déformé de $0,5 \%$ à $10 \mathrm{~K}$ et recuit à $220 \mathrm{~K}$. Cette température de recuit correspond dans ce cas au maximum de la hauteur du pic de Bordoni [7] donc à des dislocations de longueurs libres maximales.

2.4 Processus eXPérimental. - Les expériences de microfluage sont réalisées en appliquant une contrainte statique de torsion et en enregistrant la déformation de l'éprouvette. On peut opérer de deux manières schématisées par la figure 2 . Après le

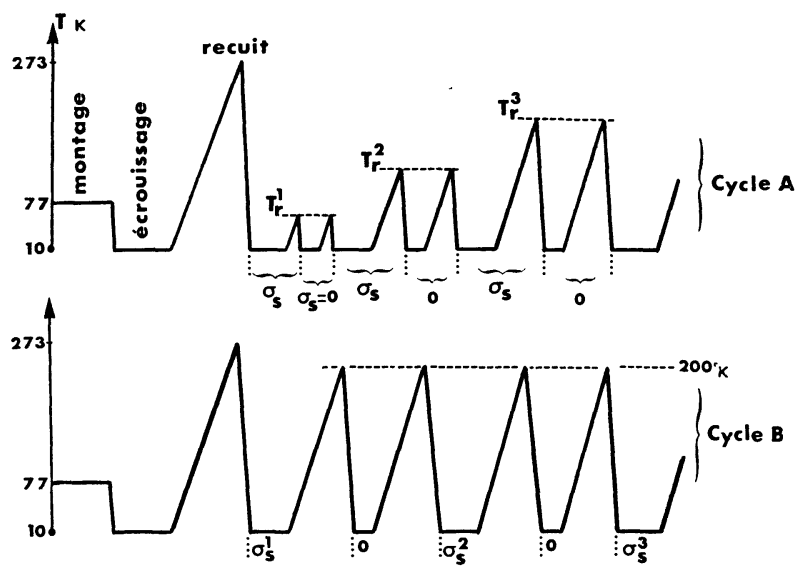

Fig. 2. - Schéma opératoire suivi pour la réalisation des expériences de microdéformation.

traitement de l'échantillon, on applique à $10 \mathrm{~K}$ une contrainte statique $\sigma_{\mathrm{s}}$, on effectue une montée en température (à $50 \mathrm{~K} / \mathrm{h}$ ) jusqu'à une température $T_{\mathrm{r}}$ puis on revient à $10 \mathrm{~K}$, température à laquelle la contrainte $\sigma_{\mathrm{s}}$ est supprimée. Le même cycle en température est alors redécrit.

On définit ainsi les deux types de cycles :

Cycles A. - L'ensemble de ces opérations est répété pour des températures $T_{\mathrm{r}}^{n}$ croissantes, la contrainte statique étant inchangée.

Cycles B. - L'ensemble de ces opérations est répété pour une température $T_{\mathrm{r}}$ constante et égale à $200 \mathrm{~K}$, la contrainte statique $\sigma_{\mathrm{s}}^{n}$ étant croissante.

Quant à la mesure de limite anélastique en torsion et à une température donnée, elle est effectuée en imposant à l'éprouvette des cycles de contrainte : croissante jusqu'à un certain niveau puis décroissante jusqu'à zéro. A chaque cycle, le niveau de contrainte est augmenté, la vitesse de déformation $\dot{\varepsilon}$ étant maintenue approximativement constante par un dispositif électronique avec une précision de l'ordre de $10 \%$.

La limite anélastique relevée correspond au niveau de contrainte appliquée lors du cycle qui donne une déformation résiduelle à contrainte nulle. On peut mesurer avec l'appareillage, des limites anélastiques correspondant à des déformations résiduelles de l'ordre de $10^{-6}$.
3. Résultats expérimentaux. - - 3.1 EXPÉRIENCES DE MICRODÉFORMATION APRÈS LE TRAITEMENT 1. 3.1.1 Cycles $A$. - La figure 3 illustre la déformation de l'éprouvette soumise à une contrainte statique $\sigma_{\mathrm{s}}$ égale à $1,3 \times 10^{-4} \mathrm{G}$ pour les températures $T_{\mathrm{r}}^{n}$ suivantes : $80-120-160-220 \mathrm{~K}$.

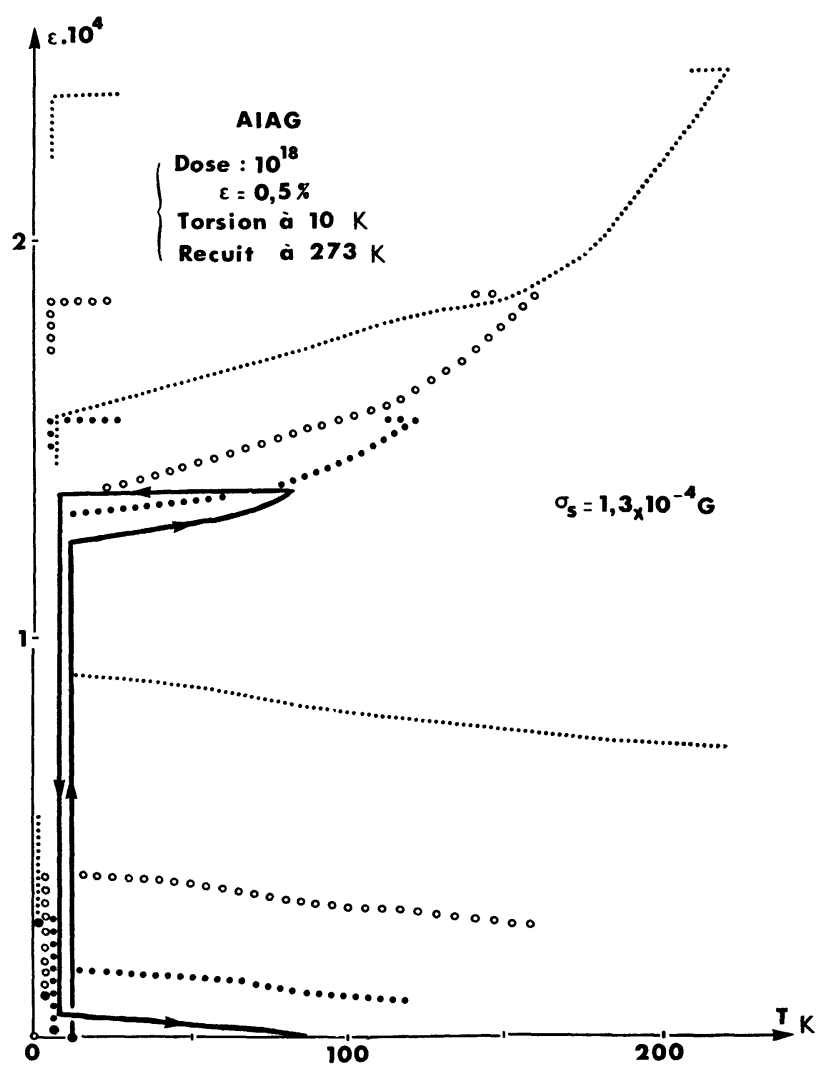

Fig. 3. - Microdéformation d'une éprouvette d'aluminium AIAG soumise au cycle $\mathrm{A}$, la contrainte statique $\sigma_{\mathrm{s}}$ est égale à $1,3 \times 10^{-4} \mathrm{G}$ et les températures $T_{\mathrm{r}}$ sont successivement 80-120-160-220 K.

La microdéformation de l'échantillon soumis à contrainte comprend deux parties : d'une part, une déformation élastique, d'autre part une déformation dépendant de la température $\left({ }^{3}\right)$. La suppression de la contrainte statique s'accompagne d'abord à $10 \mathrm{~K}$ d'un retour qui peut être plus important que la déformation élastique sous contrainte, ensuite d'une restauration thermiquement activée.

(3) L'effet de la température est prépondérant par rapport à l'effet du temps. Ceci est vérifié d'une part par le fait que l'accroissement de la déformation est très faible si on maintient la température constante (pour des temps raisonnables), d'autre part par les résultats de l'expérience décrite ci-dessous. On applique une contrainte statique et on effectue une montée en température jusqu'à $T_{\mathrm{r}}$ puis on revient à $10 \mathrm{~K}$. Sans supprimer la contrainte, on remonte à nouveau en température. Dans ces conditions, il n'y a pas d'accroissement de la déformation tant que la température $T_{\mathrm{r}}$ n'est pas atteinte : la déformation obtenue à la température $T_{\mathrm{r}}$ se maintient (aux variations de module près) au refroidissement puis au réchauffage. Lorsque la température devient supérieure à $T_{\mathrm{r}}$, la déformation se poursuit. 
Si la température $T_{\mathrm{r}}$ reste inférieure à la température du pic de Bordoni, on observe que la microdéformation est entièrement réversible à la fin d'un cycle (courbe en trait plein de la figure 3). Par contre, pour des températures $T_{\mathrm{r}}$ plus élevées, un résidu de déformation subsiste (courbe en pointillé de la figure 3).

La figure 4 montre la variation de la microdéformation thermiquement activée (courbe $B$ ) et du résidu

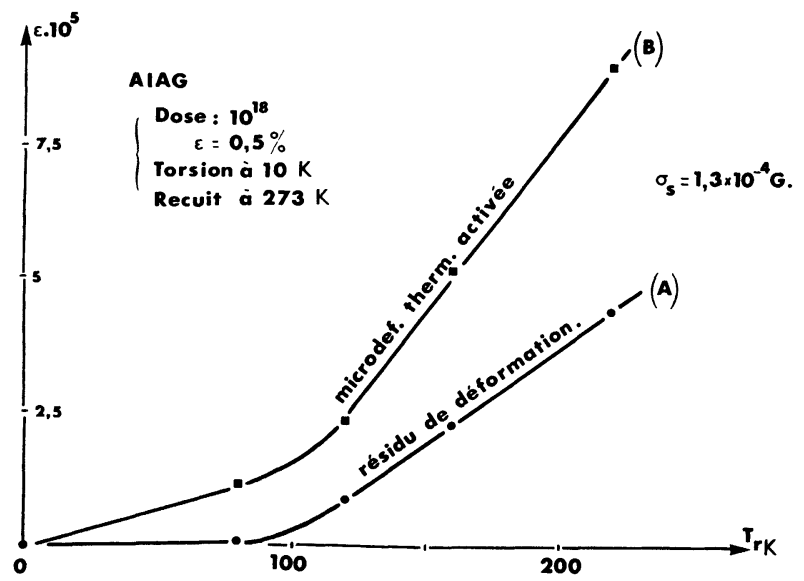

Fig. 4. - Résidu de déformation et microdéformation thermiquement activée en fonction de la température $T_{\mathrm{r}}$ atteinte lors de l'essai présenté sur la figure 3.

de déformation (courbe $A$ ) en fonction de la température de montée $T_{\mathrm{r}}$. Ces deux grandeurs augmentent rapidement au-dessus de $100 \mathrm{~K}$.

3.1.2 Cycles B. - La figure 5 montre les résultats pour trois contraintes statiques : $\sigma_{0}=0,32 \times 10^{-4} \mathrm{G}$; $\sigma_{1}=0,77 \times 10^{-4} \mathrm{G} ; \sigma_{2}=1,6 \times 10^{-4} \mathrm{G}$. Ces résultats sont semblables à ceux obtenus par Alefeld et al. [9] sur un aluminium relativement impur.
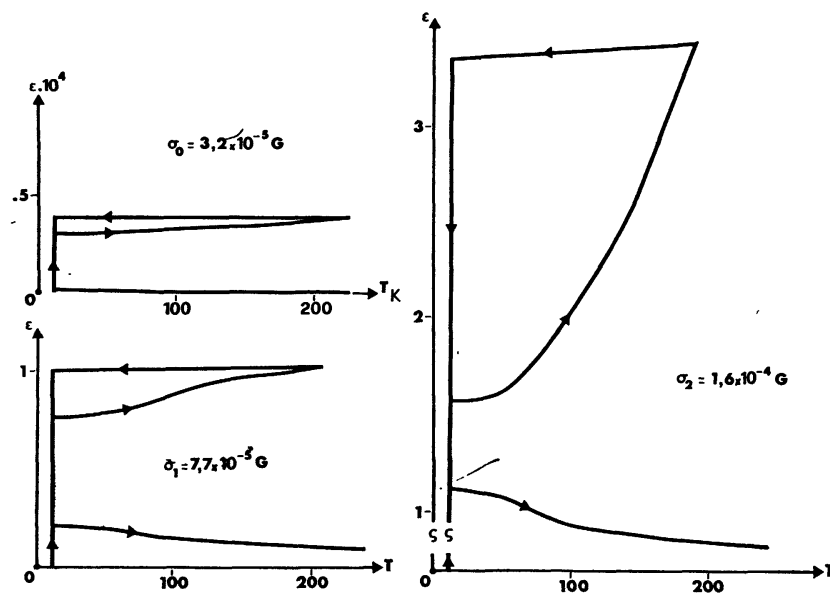

Fig. 5. - Microdéformation enregistrée sur le même aluminium déformé de $0,75 \%$ soumis au cycle $\mathrm{B}$.

Pour une faible contrainte appliquée $\left(\sigma_{0}\right)$, on peut observer que la microdéformation est pratiquement réversible quand la contrainte est supprimée à $10 \mathrm{~K}$.
Pour une contrainte statique moyenne $\left(\sigma_{1}\right)$, nous distinguons un stade de microdéformation vers $100 \mathrm{~K}$ (stade $\alpha$ ) et après suppression de la contrainte à $10 \mathrm{~K}$, il subsiste une déformation. Cette dernière est partiellement restaurée durant la montée linéaire sans contrainte (stade $\beta$ ).

Pour une contrainte plus élevée $\left(\sigma_{2}\right)$, on observe toujours un stade de microdéformation dans le domaine de température du pic de Bordoni (stade $\alpha$ ) mais à plus haute température (au-dessus de $150 \mathrm{~K}$ ), la déformation s'accroît rapidement : il pourrait s'agir d'une déformation plastique dont l'origine est différente de celle correspondant au stade $\alpha$.

Afin de mieux préciser les stades de microdéformation observés pendant les expériences précédentes, on a tracé les courbes dérivées $\mathrm{d} \varepsilon / \mathrm{d} T=f(T)$ pour les résultats correspondants à la contrainte $\sigma_{1}$ (Fig. 6) :

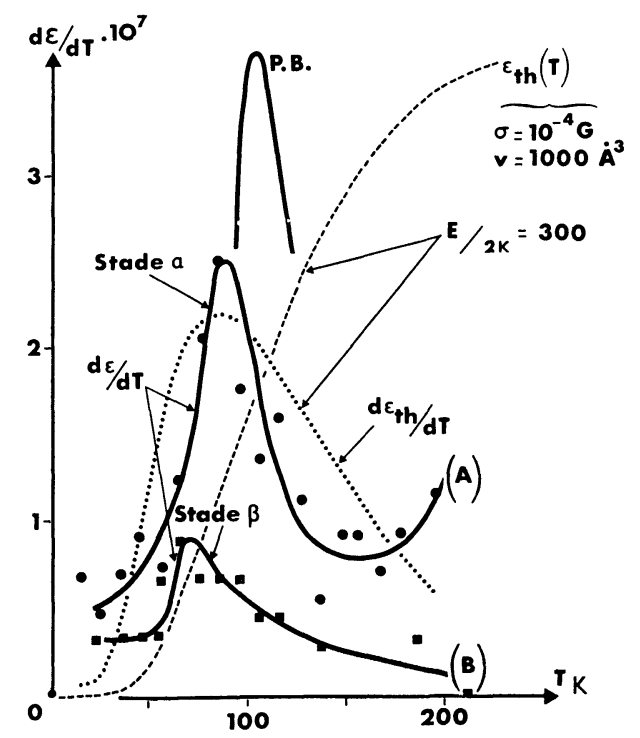

Fig. 6. - Courbes $A$ et $B$ : courbes dérivées de la microdéformation correspondant à la contrainte $\sigma_{1}$ présentée sur la figure 5 . Courbe P. B. : Position du pic de Bordoni. Courbes en pointillé : courbe donnant $\varepsilon(T)$ et sa dérivée avec la température obtenues à partir de l'expression d'Alefeld (3).

la dérivée de la microdéformation thermiquement activée présente un maximum situé vers $90 \mathrm{~K}$ (courbe $A$ ) précisant ainsi la température du stade $\alpha$; tandis que la restauration de la microdéformation est maximale vers $70 \mathrm{~K}$ (courbe $B$, stade $\beta$ ). A titre de comparaison, on montre sur la figure 6 le pic de Bordoni.

$\mathrm{Si}$ on réalise les mêmes expériences après recuit à $220 \mathrm{~K}$ (au lieu de $273 \mathrm{~K}$ ), une très faible microdéformation dans le domaine de température du pic de Bordoni est observée (Fig. 7, contrainte statique comprise entre $\sigma_{1}$ et $\sigma_{2}$ ). On sait que dans ce cas, le pic de Bordoni est très petit (voir Fig. 1).

3.2 Variation De Limite anélastiQue. - Nous avons déterminé la limite anélastique $[10,11]$ à 2 et 


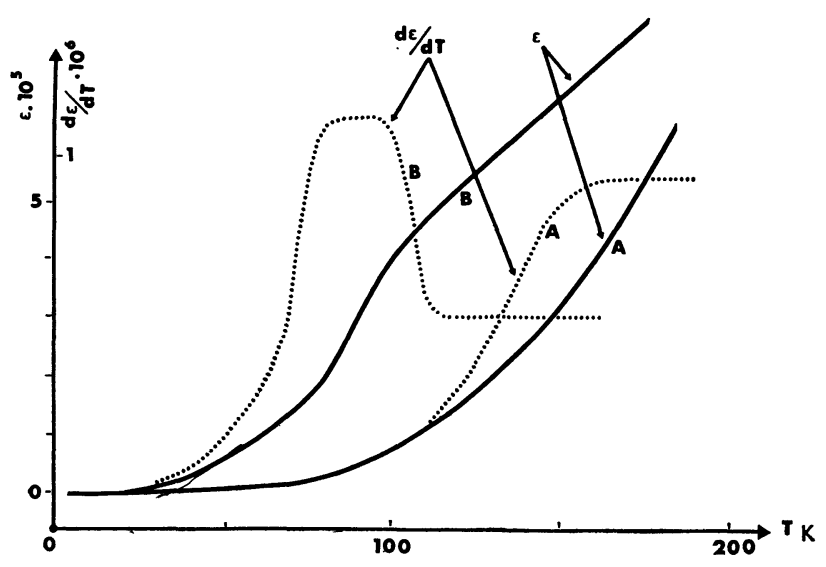

Fig. 7. - Comparaison entre les microdéformations sous contrainte statique de $10^{-4} \mathrm{G}$ (ou leurs dérivées) obtenus sur un aluminium irradié et déformé de $0,75 \%$ pour deux températures de recuit : courbes $A$ : recuit à $220 \mathrm{~K}$; courbes $B$ : recuit à $273 \mathrm{~K}$.

$5 \times 10^{-6}$ d'échantillons déformés de $0,5 \%$ à $10 \mathrm{~K}$ et recuits à $220 \mathrm{~K}$ pour deux vitesses de déformation $\left(\dot{\varepsilon}_{1}=2,5 \times 10^{-5} \mathrm{~s}^{-1}\right.$ et $\left.\dot{\varepsilon_{2}}=2,5 \times 10^{-6} \mathrm{~s}^{-1}\right)$. La figure 8 montre la variation de la limite anélastique avec la température de mesure. On observe une variation sensible de la composante thermique de la limite anélastique entre $10 \mathrm{~K}$ et $150 \mathrm{~K}$, cette variation étant plus nette au-dessous de $60 \mathrm{~K}$ environ. La composante athermique $\sigma_{\mathrm{a}}$ ne peut être définie de façon rigoureuse : la valeur choisie est celle correspondant à la limite

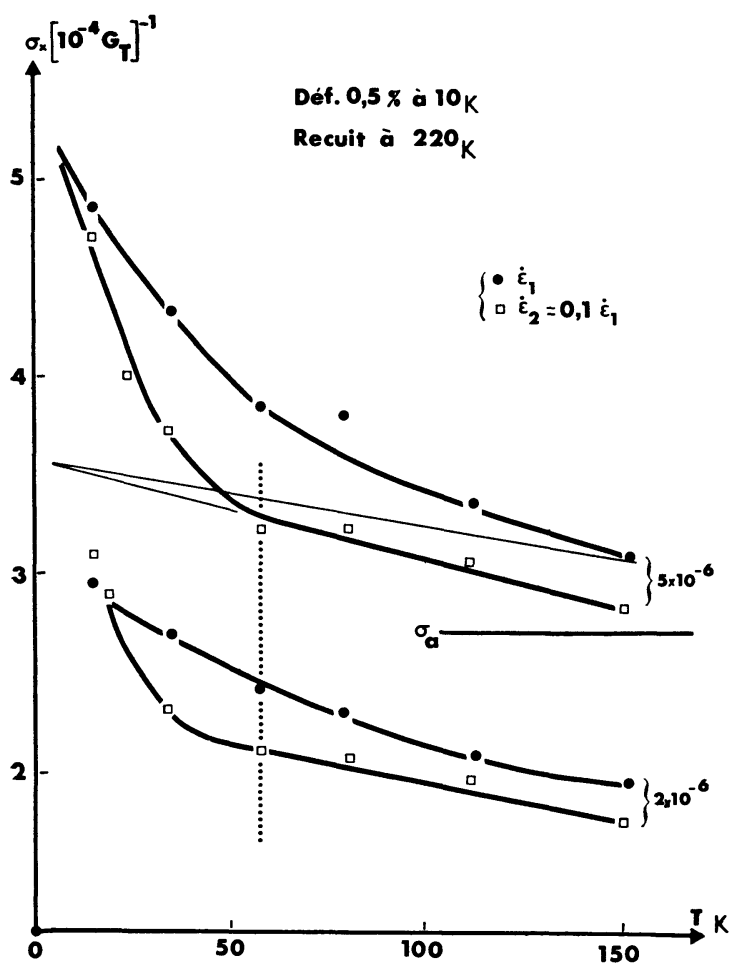

Fig. 8. - Variation de la limite anélastique à 2 et $5 \times 10^{-6}$ avec la température d'un aluminium AIAG pour deux vitesses de déformation : $\dot{\varepsilon}_{1}=2,5 \times 10^{-5} \mathrm{~s}^{-1}=10 \dot{\varepsilon}_{2}$. anélastique à $150 \mathrm{~K}$ (le niveau de la composante $\sigma_{\mathrm{z}}$ dépend de la valeur de la déformation choisie pour définir la limite anélastique).

4. Discussion des résultats. - Ainsi, nous observons avec une contrainte statique convenable une microdéformation thermiquement activée dans le domaine de température de la relaxation de Bordoni. Il est raisonnable de penser que la microdéformation et le pic de Bordoni sont dus au même mécanisme. Le modèle de la relaxation de Bordoni due à la formation thermiquement activée de doubles décrochements permet de donner une interprétation cohérente de l'ensemble des résultats dans les métaux c. f. c. [12]. Par conséquent, la microdéformation thermiquement activée peut être associée à la création et au mouvement des décrochements thermiques. Ainsi, on peut donner l'interprétation suivante des résultats présentés sur les figures 3 et 5 .

Quand on applique une contrainte statique, les dislocations tendent à atteindre leur nouvelle position d'équilibre déterminée par la tension de ligne (voir Fig. 9). Ce déplacement nécessite le franchissement de barrière de potentiel [9] dont la hauteur est comparable à l'énergie d'activation du pic de Bordoni [12].

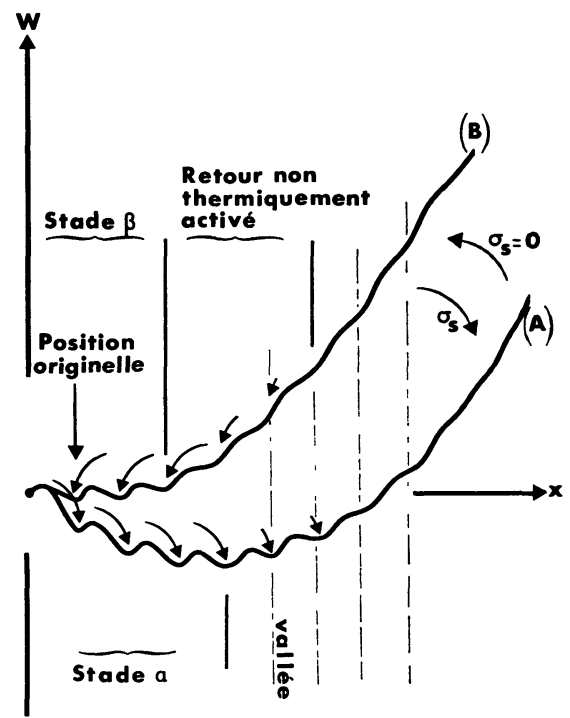

Fig. 9. - Diagramme énergétique d'une dislocation en présence de contraintes de Peierls-Nabarro en fonction du déplacement transversal $x$ aux vallées de Peierls. Courbe $A:$ la dislocation est soumise à une contrainte $\sigma_{\mathrm{s}}$. Courbe $B$ : la dislocation n'est soumise qu'à une très faible contrainte.

Nous avons déterminé le profil du diagramme énergétique d'allure parabolique (Fig. 9) d'une dislocation de longueur donnée et soumise à contrainte.

Ce calcul montre que l'énergie d'activation pour la création d'un double décrochement $E_{\mathrm{c}}$ ne dépend que de la contrainte (elle est donc indépendante de la longueur de la dislocation et du numéro de la vallée où elle se trouve) et suit approximativement 
la loi (dans l'hypothèse d'un profil sinusoïdal de la vallée de Peierls) :

$$
E_{\mathrm{c}}=2 W_{k}\left[1-\left(\frac{\sigma}{\sigma_{\mathrm{p}}}\right)^{0,63}\right]
$$

si $2 W_{k}$ est l'énergie d'un double décrochement pour $\sigma=0$. (Cette relation est issue d'un calcul effectué au laboratoire dont le principe est identique à celui utilisé par Dorn et Rajnak [13] et Arsenault [14].)

Pour les contraintes habituelles, $E_{\mathrm{c}}$ est assez voisin de $2 W_{k}$; ainsi la microdéformation thermiquement activée est observée dans le domaine de température du pic de Bordoni qui a la même énergie d'activation.

Le volume d'activation du mécanisme obtenu à partir de la relation (1) est de la forme :

$$
v^{3} \sigma \simeq \text { Cte . }
$$

On montre que ce volume est égal à l'aire balayée par la dislocation multipliée par $b\left(v_{c}\right)$ correspondant à la position de col lors de la création des paires de décrochements. Cette aire est calculée en déterminant la forme de la ligne de dislocation à partir de l'intégration de l'équation d'équilibre de la ligne [14].

L'énergie $E_{\mathrm{a}}$ et l'aire balayée multipliée par $b\left(v_{\mathrm{a}}\right)$ correspondant à la position de col pour l'annihilation des paires dépendent de la contrainte, de la longueur de la dislocation et du numéro de la vallée (voir Fig. 9). Les valeurs de $E_{\mathrm{a}}$ sont supérieures à $E_{\mathrm{c}}$ avant que la dislocation n'atteigne son minimum minimorum d'énergie, elles sont inférieures à $E_{\mathrm{c}}$ au-delà. Il existe donc une position (dépendant de la longueur et de la contrainte) où $E_{\mathrm{c}} \simeq E_{\mathrm{a}}$ (condition de Paré). Signalons enfin que le volume $v_{\text {a }}$ est beaucoup plus important que $v_{\mathrm{c}}$ (d'un ordre de grandeur).

Lors de la suppression de la contrainte, les dislocations tendent à revenir vers leur position initiale mais pour ce faire doivent franchir les barrières $E_{\mathrm{a}}$. A $0 \mathrm{~K}$, sous contrainte nulle, seuls sont possibles les sauts pour lesquels $E_{\mathrm{a}}=0$. Ainsi à $10 \mathrm{~K}$, on observe un retour élastique plus important que la déformation élastique lors de l'application de la même contrainte (cf. Fig. 3).

Les sauts pour lesquels $E_{\mathrm{a}}$ n'est pas nulle, sont responsables de la restauration observée lors de la montée linéaire sans contrainte. Les valeurs de $E_{\mathrm{a}}$ associées à cette restauration sont comprises entre 0 et $E_{\mathrm{c}}$ (Fig. 9), ainsi se produira-t-elle plus tôt en température (stade $\beta$ ) que la déformation elle-même (stade $\alpha, E_{\mathrm{c}} \simeq 2 W_{k}$ ) (cf. Fig. 6).

A l'aide du schéma précédent, on comprend alors l'évolution de la microdéformation thermiquement activée et du résidu de déformation avec la température de montée $T_{\mathrm{r}}$ (Fig. 4). En effet, les dislocations ne deviennent suffisamment mobiles que dans le domaine de température du pic de Bordoni.

On peut déterminer les valeurs de $E$ et $v$ à $l$ 'aide des résultats précédents. En effet, la déformation anélasti- que due à la création et au mouvement des décrochements thermiques a été calculée par Alefeld et al. [15] $\left({ }^{4}\right)$. Dans le cas de dislocations parallèles aux vallées de Peierls et en négligeant l'interaction des décrochements, on a :

$$
\varepsilon(\sigma, T)=\Lambda a L \exp \left(\frac{-E}{2 k T}\right) \frac{\operatorname{sh} u}{u} L(u)
$$

avec

$$
u=\frac{v \sigma}{2 k T} \text { et } L(u) \text { fonction de Langevin }
$$

1: densité des dislocations,

$a$ : paramètre du réseau,

$b$ : vecteur de Burgers,

$L$ : longueur des boucles,

$E$ : énergie d'activation du mécanisme : $E=E_{\mathrm{c}}\left(\sigma_{\mathrm{i}}\right)$,

$v$ : volume d'activation,

$\sigma_{\mathrm{i}}$ : contrainte interne locale sur la ligne.

La fonction de Langevin décrit le mouvement latéral des décrochements tandis que l'autre terme représente la création des paires.

L'allure de la courbe théorique $\varepsilon_{\mathrm{th}}(T)$ est tout à fait comparable à la forme des courbes expérimentales tant que la contrainte n'est pas trop élevée $\left(<\sigma_{2}\right)$ (cf. Fig. 6). La relation (3) présentant un accord qualitatif avec les résultats expérimentaux, on peut déterminer les ordres de grandeur pour $E$ et $v$. En effet, dans l'hypothèse d'une énergie et d'un volume d'activation indépendants de la contrainte, un premier calcul peut être fait en recherchant les couples de températures qui gardent $u$ constant, soit :

$$
\frac{\sigma_{0}}{T_{0}}=\frac{\sigma_{1}}{T_{1}}=\frac{\sigma_{2}}{T_{2}}
$$

On a alors :

$$
\ln \frac{\varepsilon\left(\sigma_{1}, T_{1}\right)}{\varepsilon\left(\sigma_{2}, T_{2}\right)}=\frac{E}{2 k}\left(\frac{1}{T_{2}}-\frac{1}{T_{1}}\right) .
$$

Les valeurs de $E$ déduites de la relation (4) sont reportées dans le tableau I, ligne $a$.

Si l'on veut tenir compte de la variation de $E$ et $v$ avec la contrainte (relations (1) et (2)), $u$ est constant si :

$$
\frac{T_{1}}{T_{2}}=\left(\frac{\sigma_{1}}{\sigma_{2}}\right)^{2 / 3}
$$

on obtient alors les résultats indiqués par le tableau I, ligne $b$.

(4) Cette relation a été établie dans des conditions d'équilibre thermique. On peut considérer que c'est presque le cas dans nos conditions expérimentales (vitesse de montée relativement faible). On vérifie la validité de cette remarque en stoppant l'évolution de la température, la microdéformation ne se poursuit pratiquement pas. 
Ces résultats sont assez proches des précédents.

\begin{tabular}{crrrrrr} 
& \multicolumn{7}{c}{ TABLEAU I } \\
& $T_{1}$ & 54 & 64 & 75 & 81 & 103 \\
& $T_{2}$ & 100 & 120 & 140 & 150 & 190 \\
(a) & $E / k$ & 706 & 784 & 946 & 1004 & 1240 \\
(b) & $E / k$ & 622 & 720 & 810 & 888 & 1226 \\
& $T$ & 70 & 100 & 120 & 150 & \\
& $v / b^{3}$ & 54 & 86 & 111 & 172 &
\end{tabular}

La détermination du volume d'activation se fait par la résolution de l'équation :

$$
\frac{\varepsilon\left(\sigma_{2}, T\right)}{\varepsilon\left(\sigma_{1}, T\right)}=\left(\frac{u_{1}}{u_{2}}\right)^{2} \frac{u_{2} \operatorname{ch} u_{2}-\operatorname{sh} u_{2}}{u_{1} \operatorname{ch} u_{1}-\operatorname{sh} u_{1}}
$$

la déformation étant mesurée à la température $T$ et pour

$$
\frac{u_{2}}{u_{1}}=\frac{\sigma_{2}}{\sigma_{1}}
$$

(on suppose que $E$ et $v$ sont indépendants de $\sigma$ ).

Les résultats obtenus sont reportés dans le tableau I.

La figure 10 montre la variation de $E$ avec la température $\left(T_{1}+T_{2}\right) / 2$ obtenue à partir des résultats précédents.

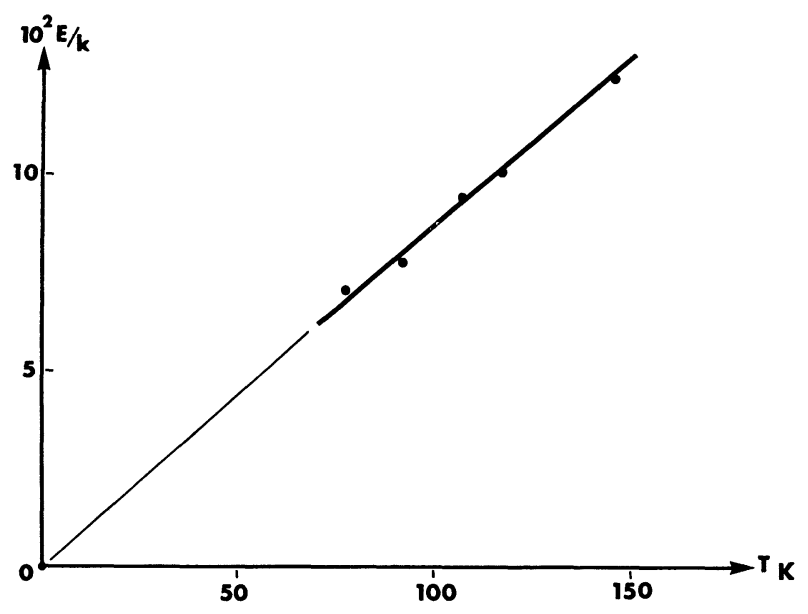

Fig. 10. - Variation de l'énergie d'activation $E$ obtenue à partir de la relation (4) en fonction de la température.

On observe que $E$ est de la forme :

$$
E=\alpha k T \text { avec } \alpha \simeq 8 .
$$

A partir de la valeur moyenne de $E(E / k \simeq 800)$, on peut déterminer

$$
2 W_{k} \simeq 0,1 \mathrm{eV}\left(\text { avec } \sigma_{\mathrm{p}} \simeq 5 \times 10^{-4} \mathrm{G}\right),
$$

valeur proche de celle déduite de l'énergie d'activation du pic de Bordoni [2]. D'autre part, le produit $\Lambda L$ peut être calculé à partir de la relation (3) : on trouve

$$
\Lambda L \simeq 2,5 \times 10^{7} \mathrm{~m}^{-1}
$$

soit

$$
2500 \AA<L<25000 \AA
$$

pour

$$
10^{13}<\Lambda<10^{14} \mathrm{~m}^{-2},
$$

ordre de grandeur tout à fait correct.

Ainsi, les expériences de microfluage semblent montrer qu'il existe dans le domaine de température du pic de Bordoni une microdéformation associée à la création thermiquement activée de doubles décrochements. La valeur moyenne du volume d'activation $\left(\simeq 90 b^{3}\right)$ est compatible avec la valeur théorique $2 \omega b^{2}, \omega$ étant la largeur d'un décrochement ( $\omega$ est de l'ordre de $50 b$ dans les c. f. c.). L'énergie d'activation du mécanisme croît linéairement avec la température : on a donc une distribution des valeurs de $E$ qui est due à une répartition des contraintes internes.

Les mêmes expériences effectuées sur un aluminium ayant subi le traitement 2 , donnent des résultats très semblables aux précédents : l'amplitude de la microdéformation est seulement plus faible (à contrainte statique identique) ainsi que les valeurs de $E$ et $v$ $\left(E \simeq 0,05 \mathrm{eV}\right.$ et $\left.v \simeq 50 b^{3}\right)$. Ceci est probablement dû à l'effet de contraintes internes plus élevées (le pic de Bordoni est alors bien défini, la condition de Paré étant remplie).

Afin de vérifier que ces résultats ne sont pas liés à la pureté du matériau, les mêmes expériences ont été effectuées sur un aluminium obtenu par zone fondue, après les deux traitements 1 et 2 . Dans ce cas une microdéformation thermiquement activée est également détectée ainsi qu'une restauration de la microdéformation sous contrainte nulle.

Si l'on veut mettre en évidence l'effet des barrières de Peierls, il faut mesurer la limite anélastique correspondant aux tous premiers mouvements de dislocations car dans le cas contraire, le mécanisme d'intersection va masquer le mécanisme de Peierls. Nos expériences, où l'on détecte la limite anélastique à partir de quelque $10^{-6}$ satisfont à cette condition.

A partir des résultats de la figure 8 , on peut déterminer les paramètres d'activation et leur variation avec la contrainte ou la température, à savoir l'enthalpie d'activation et le volume d'activation définis par [16] :

$$
\begin{aligned}
\Delta H & =-k T^{2}\left(\frac{\partial \ln \dot{\varepsilon}}{\partial \sigma^{*}}\right)_{T}\left(\frac{\partial \sigma^{*}}{\partial T}\right) \varepsilon \\
v^{*} & =k T\left(\frac{\partial \ln \dot{\varepsilon}}{\partial \sigma^{*}}\right)_{T}
\end{aligned}
$$

où $\sigma^{*}=$ contrainte effective $=\left(\sigma-\sigma_{\mathrm{a}}\right) / 2$,

$\sigma=$ contrainte appliquée,

$\sigma_{\mathrm{a}}=$ composante athermique de la contrainte.

On peut aussi calculer l'enthalpie d'activation 
totale $H_{0}$ (enthalpie d'activation $\Delta H$ à contrainte effective $\sigma^{*}$ nulle) :

$$
H_{0}=\Delta H+\int_{0}^{\sigma^{*}} v^{*} \mathrm{~d} \sigma^{*}
$$

si l'on admet que l'entropie varie lentement avec la contrainte ou la température.

La figure 11 montre la variation de $v^{*}$ avec $\sigma^{*}$ et de $\Delta H$ et $H_{0}$ avec la température $\left({ }^{5}\right)$ obtenus à partir de la mesure de la limite anélastique à $5 \times 10^{-6}$.
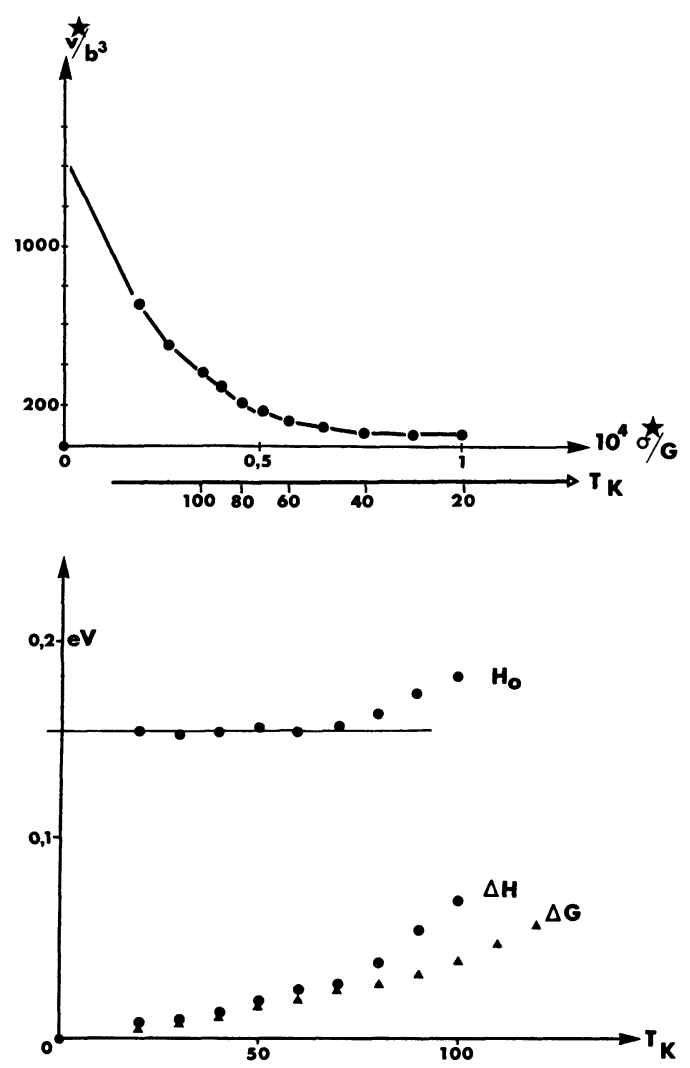

Fig. 11. - Variation avec la température ou la contrainte effective $\sigma^{*}$ du volume d'activation apparent $v^{*}$, de l'enthalpie d'activation $\Delta H$, de l'enthalpie d'activation totale $H_{0}$ et de l'énergie libre $\Delta G$.

On peut s'affranchir du choix de la composante athermique $\sigma_{\mathrm{a}}$ en supposant qu'un autre mécanisme se superpose au premier vers les hautes températures (comme le laisserait supposer la variation linéaire de $\sigma(T)$ à $\dot{\varepsilon}_{2}$ au-dessus de $\left.60 \mathrm{~K}\right)$. Ce mécanisme pourrait correspondre à un mécanisme d'intersection [3]. En reprenant l'analyse précédente après avoir soustrait les parties linéaires des courbes $\sigma(T)$ de la figure 8 , on obtient des résultats très proches de ceux présentés sur la figure 11 .

(5) La valeur de $H_{0}$ dépend du choix de la composante $\sigma_{\mathrm{a}}$; cette dernière n'étant pas définie de façon rigoureuse, on ne peut donc déterminer en absolu $H_{0}$ avec une grande précision mais son ordre de grandeur est toujours correct.
En outre, la détermination de l'énergie libre de Gibbs d'activation du mécanisme $\Delta G$ :

$$
\Delta G=\Delta H-T \Delta S \simeq-\int_{\sigma_{0}^{*}}^{\sigma^{*}} v^{*} \mathrm{~d} \sigma^{*}
$$

(si l'on ne tient pas compte de la variation du module $G$ avec la température) où $\sigma_{0}^{*}$ est la contrainte effective à $0 \mathrm{~K}$, montre que $\Delta G$ et $\Delta H$ (Fig. 11$)$, sont très voisins à basse température. Au-delà l'importance du terme entropique se fait sentir; ainsi les résultats obtenus par les deux méthodes sont concordants.

On constate que dans le domaine de température 20-80 $\mathrm{K}$, les valeurs de $\Delta H$ et $v^{*}$ sont tout à fait comparables à celles trouvées précédemment soit $E$ et $v$, renforçant ainsi la validité de notre hypothèse. $H_{0}$ étant indépendant de la température (jusque vers $80 \mathrm{~K})$, le même mécanisme contrôle donc le mouvement des dislocations jusqu'à cette température au moins.

Enfin si on tient compte des relations (6) et (7), on a :

$$
\Delta H=-v^{*} T\left(\frac{\partial \sigma^{*}}{\partial T}\right)_{\dot{\varepsilon}} \simeq \Delta G=\alpha k T
$$

avec

$$
\alpha=\ln \frac{\dot{\varepsilon}_{0}}{\dot{\varepsilon}}=-\frac{v^{*}}{k}\left(\frac{\partial \sigma^{*}}{\partial T}\right)_{\dot{\varepsilon}}=\ln \frac{b v_{\mathrm{D}}}{V}
$$

où

$$
\dot{\varepsilon}_{0} \simeq \Lambda b^{2} v_{\mathrm{D}}, \quad \dot{\varepsilon}=\Lambda b V,
$$

$V=$ vitesse de déplacement des dislocations.

La valeur de $\alpha$ déterminée à partir des résultats de la figure 11 est de l'ordre de 7 (valeur proche de celle obtenue par l'analyse des courbes de microdéformation sous contrainte constante). Habituellement, les valeurs du paramètre $\alpha$ sont voisines de 25 [17]. Notre résultat signifie donc que les dislocations sont très mobiles (10) ou que le processus de déformation a une énergie d'activation et un volume d'activation faibles, ce qui est caractéristique d'un mécanisme de création de doubles décrochements dans les métaux c. f. c. (la contrainte de Peierls y est faible). On peut d'ailleurs remarquer que dans le cas des c. c. où la contrainte de Peierls est environ dix fois supérieure à celle des c. f. c., le terme $\left(\frac{\partial \sigma^{*}}{\partial T}\right)_{\dot{\varepsilon}}$ est multiplié par 10 alors que $v=2 \omega b^{2} \propto \sigma_{\mathrm{p}}^{-1 / 2}$ [19] est donc divisé par $\sqrt{10}$. En conséquence, le paramètre $\alpha$ dans les c. c. doit être proche de $7 \sqrt{10} \simeq 22$ ce qui est bien observé expérimentalement [20].

5. Conclusion. - La relaxation de Bordoni dans les métaux c. f. c. est généralement attribuée à la formation thermiquement activée de doubles décrochements (modèle de Seeger-Paré). A partir des carac- 
téristiques du pic de Bordoni, on peut calculer la contrainte de Peierls-Nabarro, le mouvement des dislocations parallèles ou presque aux rangées denses étant contrôlé par l'existence des barrières de Peierls.

Un tel mouvement entraîne une déformation anélastique réversible thermiquement activée dans le domaine de température du pic de Bordoni. Les résultats obtenus confirment cette hypothèse de l'analyse de la microdéformation observée; on détermine l'énergie et le volume d'activation (de l'ordre de $0,1 \mathrm{eV}$ et $100 b^{3}$ respectivement), qui sont tout à fait compatibles avec le mécanisme de création de doubles décrochements. De plus, l'étude de la variation de la limite anélastique avec la température et la vitesse de déformation confirme l'existence d'un tel mécanisme.

Remerciements. - Nous tenons à remercier M. Bonjour du C. E. N. Grenoble (P. C. B. T.) grâce à qui l'irradiation des échantillons a été effectuée ainsi que M. Revel du Centre d'Etudes de Chimie Métallurgique de Vitry/Seine qui nous a procuré l'aluminium de zone fondue.

Nos remerciements vont également à M. Kubin du Centre de Microscopie Electronique du CNRS de Toulouse pour ses pertinentes remarques.

\section{Bibliographie}

[1] Nowick, A. S. et Berry, B. S., Anelastic Relaxation in Cristalline Solids (Academic Press, New York) 1972.

[2] Seeger, A., J. Physique Colloq. 32 (1971) C2-193.

[3] Mitra, S. K., DoRn, J. E., Trans. AIME 227 (1963) 1015.

[4] Friedel, J., Dislocations (Pergamon Press, New York) 1964.

[5] Seeger, A., Mader, S. et Kronmüller, H., Electron Microscopy and Strength of Crystals (Interscience Publishers, New York) 1963, 665.

[6] FRIEDEL, J., Electron Microscopy and Strength of Crystals (Interscience Publishers, New York) 1963, 605.

(7] Esnouf, C., Fantozzi, G. et Gobin, P., Mem. Sci. Rev. Met. 10 (1974) 637.

[8] Pare, V. K., J. Appl. Phys. 3 (1961) 332.

[9] Alefeld, G., Filloux, J. et Harper, H., Dislocation Dynamics (Mc Graw Hill, New York) 1968, 191.

[10] Brown, N. et Ekvall, R. A., Act. Met. 10 (1962) 1101.
[11] Boudet, A., Thèse, Université Paul-Sabatier de Toulouse. [12] Benort, W., Fantozzi, G. et Esnouf, C., Second European Conference on Internal Friction and Ultrasonic Attenuation in Solids, Rome, septembre 1975.

[13] Dorn, J. E. et RajnaK, S., Trans. AIME 230 (1964) 1052.

[14] Arsenault, R. J., Act. Met. 15 (1967) 501.

[15] Alefeld, G., Chambers, R. H. et Firle, T. G., Phys. Rev. 140 (1965) A 1771.

[16] Evans, A. G. et Rawlings, R. D., Phys. Stat. Solids 34 (1969) 9.

[17] Kubin, L., Phil. Mag. 30 (1974) 4, 705.

[18] Cagnon, M., Thèse, Université Paris-Sud, 1973.

[19] HiRTH, J. P. et LotHe, J., Theory of dislocations (Mc GrawHill, New York) 1968.

[20] Conte, R., Groh, P. et Escaig, B., Phys. Stat. Solids 28 (1968) 475. 